\title{
Diacronie
}

Studi di Storia Contemporanea

$N^{\circ} 7,3 \mid 2011$

«Spagna Anno Zero»: la guerra come soluzione

\section{Barcellona, 17 e 18 marzo 1938}

\section{Edoardo Grassia}

\section{(2) OpenEdition \\ Journals}

\section{Edizione digitale}

URL: http://journals.openedition.org/diacronie/3237

DOI: $10.4000 /$ diacronie.3237

ISSN: 2038-0925

\section{Editore}

Association culturelle Diacronie

\section{Notizia bibliografica digitale}

Edoardo Grassia, «Barcellona, 17 e 18 marzo 1938 », Diacronie [Online], № 7, 3 | 2011, documento 6 ,

Messo online il 29 juillet 2011, consultato il 01 mai 2019. URL : http://journals.openedition.org/

diacronie/3237 ; DOI : 10.4000/diacronie.3237 


\section{Diacronie}

N. 7 | 7|2011 Spagna Anno Zero: la guerra come soluzione

\section{6/}

\section{Barcellona, 17 e 18 marzo 1938}

Edoardo GRASSIA *

Il 17 e 18 marzo 1938 Barcellona subì pesantissimi bombardamenti ad opera di aerei dell'Aviazione Legionaria decollati da Palma di Maiorca. Ciano riferì all'ambasciatore inglese che l'ordine di bombardare era partito direttamente da Franco, ma in realtà lo diede Mussolini al Generale Valle, incontrato casualmente alla Camera dove il Duce italiano si era recato per fare un atteso discorso circa l'Anschluss. Perché quest'ordine? È stato più volte scritto che le motivazioni vanno cercate nella volontà di fiaccare il morale della popolazione civile, secondo i migliori insegnamenti della dottrina aerea del Douhet, o nella risposta alla riapertura della frontiera francese per il transito degli aiuti alla fazione repubblicana. Queste motivazioni, per quanto valide, si sono concentrate solo all'interno del conflitto civile spagnolo. Nel mio articolo, in un ottica che prende in considerazione l'approssimarsi della Seconda Guerra Mondiale, cercherò di argomentare un'ulteriore motivazione proprio a partire dall'annessione dell'Austria realizzata dai tedeschi e dalla volontà dichiarata di Mussolini di accrescere le sue quotazioni presso Hitler in un'ottica di futura alleanza militare. I bombardamenti di Barcellona del 17 e 18 marzo 1938, da questo punto di vista, acquisiscono una dimensione comunicativa di tenacia, spregiudicatezza e potenza militare, che, almeno in apparenza, il Duce, cavalcando l'onda euforica innescata dall'Anschluss, voleva trasmettere al possibile alleato. 


\section{Premessa}

apoluogo della regione della Catalogna e sede del Governo repubblicano
trasferitosi da Valencia il 31 ottobre 1937; punto di raccolta delle Brigate
Internazionali e centro di scontri armati tra anarchici e comunisti;
fondamentale porto per lo sbarco di materiale bellico in aiuto della fazione repubblicana e punto focale degli attacchi aerei della parte nazionalista: la città di Barcellona potrebbe essere considerata uno dei luoghi simbolo della guerra civile spagnola.

12 Marzo 1938: l'attenzione internazionale si distrae dalla Spagna e si rivolge all'Austria: Hitler realizza l'Anschluss. Cinque giorni dopo aerei dell'Aviazione Legionaria bombardano pesantemente il capoluogo catalano. Perché tale ordine? Per infliggere un colpo risolutivo che determinasse il crollo del regime repubblicano e per rispondere alla riapertura della frontiera francese e al conseguente transito di aiuti agli oppositori di Franco. Probabilmente, però, le cause vanno cercate anche a partire da ciò che accade in Austria e dalla euforica lettura che di tale evento fece Mussolini. Questi infatti, dovendo pronunciare un discorso alla Camera dei Deputati attinente all'azione tedesca, incontrò in maniera fortuita il Capo di Stato Maggiore della Regia Aeronautica, anch'egli lì per esporre all'alto consesso lo stato della forza armata aerea. Un incontro veloce quanto basta per impartire l'ordine di colpire violentemente Barcellona con gli aerei stazionati a Palma di Maiorca. Ne scaturì un triste preludio di ciò che vissero molte città europee durante la Seconda Guerra Mondiale; basti considerare che gli ambienti militari americani, a seguito dei bombardamenti effettuati sulla città di Roma il 19 luglio 1943, dissero che era stato «un bombardamento di successo» usando, nell'occasione, le stesse parole pronunciate da Ciano dopo il bombardamento di Barcellona ${ }^{1}$.

\section{Introduzione}

Tra l'Ottocento e il Novecento, grazie alla sua posizione geografica affacciata sul Mediterraneo, Barcellona si sviluppò come uno dei più importanti porti europei, divenne uno dei principali centri industriali spagnoli, affermandosi come punto di collegamento interno ed internazionale. Tale sviluppo portò, nel primo trentennio del

1 Disponibile al sito URL: < http://cronologia.leonardo.it/storia/a1943a.htm > [consultato in data 
Novecento, a raddoppiare i suoi abitanti che passarono da circa 533 mila dell'inizio secolo a poco oltre un milione durante gli anni Trenta. E proprio su di essi furono effettuati i drammatici bombardamenti della guerra civile. Florido centro economico, Barcellona fu anche un importantissimo centro di propulsione culturale. Limitandoci ai nomi abbiamo l'urbanista Cerdà, gli architetti Eusebi Guell e Antoni Gaudi, i pittori Montaner, Picasso che giunse in città a 13 anni, Mirò e ancora Casas e Dalì che proprio a Barcellona propose, nel 1925, la sua prima mostra personale: fu proprio dalla ciudad condal che, nel settembre 1923, il generale Miguel Primo de Rivera partì - in qualità di Capitano Generale - nella sua personale "marcia su Madrid" per ottenere dalle mani del re Alfonso XIII il controllo del Governo.

Allo scoppio del conflitto civile del 1936, Barcellona, così come la maggior parte delle principali città spagnole, rimase fedele al Governo in carica divenendo, il 31 ottobre 1937 e fino alla sua occupazione da parte delle forze nazionaliste avvenuta il 26 gennaio 1939, sede del Governo Repubblicano di Negrin. Il primo atto politico di Franco nel territorio appena conquistato fu quello di abolire l'autonomia catalana. Anche la lingua catalana, propria della regione, fu messa al bando e sostituita con quella castigliana ${ }^{2}$. Nei giorni successivi la caduta della città, in migliaia fuggirono verso un difficile esilio in Francia, mentre molti altri furono giustiziati durante la rappresaglie delle forze nazionaliste.

\section{Aviazione Legionaria delle Baleari: $8^{\circ}$ Stormo Bombardamento Veloce "Falchi delle Baleari" e XXV Gruppo Autonomo Bombardamento Notturno "Pipistrelli delle Baleari"}

Sono ormai molte e quasi tutte storicamente note le azioni di bombardamento aereo su città densamente abitate che hanno trovato attuazione durante i conflitti del XX secolo. Ancor prima della guerra civile spagnola, teorici di strategie aeree come Billy Mitchell, Sir Hugh Trenchard e, soprattutto, Giulio Douhet, sostennero il bombardamento strategico, ovvero, "una azione di guerra che si svolge lontano dai campi di battaglia per andare a colpire, tra l'altro, anche le città". La teoria del Douhet, secondo cui «immaginiamoci una grande città che, in pochi minuti, veda la sua parte centrale, per un raggio di 250 metri all’incirca, colpita da una massa di proiettili dal peso complessivo di una ventina di tonnellate: qualche esplosione, qualche principio di incendio, gas venefici che uccidono e impediscono di avvicinarsi alla zona colpita: poi

2 BROWN, Harry, La guerra civile spagnola, Bologna, II Mulino, 2000, p. 120. 
gli incendi che si sviluppano, il veleno che permane; passano le ore, passa la notte, sempre più divampano gli incendi, mentre il veleno filtra e allarga la sua azione. La vita della città è sospesa; se attraverso ad esse passa qualche grossa arteria stradale, il passaggio è sospeso»3, aveva più di un decennio e godeva ormai di un certo consenso internazionale.

Durante la Prima Guerra Mondiale velivoli Avro 504 e Royal Air Navy Service bombardarono Colonia e Düsseldorf, inaugurando la lunga e tragica serie dei bombardamenti nelle città. Il Generale inglese Ian Smuts profeticamente scrisse «non è lontano il tempo in cui le operazioni aeree, con la devastazione del territorio nemico e la distruzione dei centri urbani su vasta scala, diventeranno le principali azioni di guerra alle quali la vecchia forma di operazioni terrestri e navali saranno subordinate perché secondarie»4. Tutto ciò ci fa comprendere come il clima culturale prevalente negli ambienti militari internazionali non avesse ancora alcuna traccia di quel Diritto Internazionale Umanitario che prenderà corpo solo dopo il Secondo Conflitto Mondiale alla luce delle tragiche molteplici esperienze, tra cui, anche quelle spagnole.

Proprio in Spagna, sul versante nazionalista, si schierarono tre forze aeree alleate: la Legione Condor tedesca, l'Aviazione Legionaria italiana e la Jefatura del Aire spagnola. L'Aviazione Legionaria, ovvero gli Stormi di volo della Regia Aeronautica italiana che, sotto mentite spoglie combatterono a fianco dei nazionalisti del Generale Francisco Franco, fu divisa in due parti: l'Aviazione Legionaria Continentale, con sede a Vitoria, e l'Aviazione Legionaria delle Baleari, con sede a Palma di Maiorca. Mentre la prima riceveva gli ordini dal Comando del Corpo Truppe Volontarie (poi Missione Militare Italiana in Spagna - MMIS), la componente delle Baleari riceveva spesso i suoi comandi direttamente da Mussolini e Ciano, così come rivelano le pagine del diario personale del Ministro degli Esteri5. E fu proprio l'Aviazione Legionaria delle Baleari che, come vedremo, colpì ripetutamente la città di Barcellona con le azioni dei suoi bombardieri.

A Palma di Maiorca, come reparti da bombardamento aereo, furono schierati l's ${ }^{\circ}$ Stormo Bombardamento Veloce e il XXV Gruppo Autonomo Bombardamento Notturno $^{6}$. Entrambi, come tutti quelli che operarono in Spagna, riportarono su dei

3 DOUHET, Giulio, I/ dominio dell'aria e altri scritti, Roma, Aeronautica Militare Ufficio Storico dello Stato Maggiore, 2002, p. 187.

4 PELLICCIA, Antonio, "Riflessioni storiche sul potere aereo», Aeronautica, n. 3, Marzo 2007, pp. 8-11.

5 CIANO, Galeazzo, Diario 1937-1943, cit. Nel testo si incontrano molto spesso delle indicazioni che Ciano o Mussolini diedero ai comandanti territoriali in Spagna sulla condotta della guerra. Per I'Aviazione Legionaria si vedano in particolare pp. 92, 95, 96, 104 e 115.

6 GRASSIA, Edoardo, L'Aviazione Legionaria da bombardamento - Spagna 1936-1939 - Iniziare da stanotte azione violenta su Barcellona, Roma, IBN Editore, 2009, pp. 131 - 155. 
documenti ufficiali, nominati "diari storici", tutte le attività svolte giornalmente, nonché altre notizie come il numero e il tipo di aerei efficienti, l'organico del personale in forza e i relativi trasferimenti in entrata e uscita, le onorificenze assegnate e, naturalmente, la sommaria descrizione dei voli con i quali furono effettuate le azioni di guerra e di pace7.

Il diario storico dell' $8^{\circ}$ Stormo fornisce informazioni sugli avvenimenti giornalieri dal 12 gennaio 1938 sino al 30 marzo 1939, oltre a notizie sommarie mensili per il periodo aprile-maggio 1939. In apertura del documento, con caratteri maiuscoli, è scritto: «CAMPAGNA PER L'AFFERMAZIONE DELL'IDEA FASCISTA», mentre, nella premessa, si legge: «L'8 ${ }^{\circ}$ STORMO, AVUTO L'AMBITO ONORE DI POTER PRESTARE OPERA IN TERRA DI SPAGNA PER LA DIFESA DI UN SUPREMO IDEALE, SI TRASFERIVA IN DUE SUCCESSIVE SPEDIZIONI, CON I SUOI DUE GRUPPI (IL $27^{\circ}$ E IL $28^{\circ}$ ), SULLA BASE DI PALMA DE MALLORCA OVE SI RICOSTITUIVA IN DATA 12 GENNAIO 1938 XVI. FORTE DELLA ACCURATA PREPARAZIONE DI PACE, SICURO DEI SUOI MEZZI MATERIALI E MORALI, AVVALENDOSI DELLA BREVE ESPERIENZA DI GUERRA DEL $28^{\circ}$ GRUPPO, SUO PRIMO REPARTO DISTACCATO ALLE BALEARI, INIZIAVA LA CAMPAGNA DOPO DUE GIORNI DI VISITA SULLA NUOVA BASE, CON LA CERTEZZA CHE LA VITTORIA NON SAREBBE MAI MANCARA AD ESSO».

Per quanto concerne invece il XXV Gruppo Autonomo "Pipistrelli delle Baleari", il diario storico ci rende conto della sua esistenza dal 5 maggio 1938, per cui sarà considerato nella nostra illustrazione dei fatti del marzo 1938 prendendo spunto dai documenti relativi alle Squadriglie che lo componevano.

\section{2. "Marzo 1938": i bombardamenti, il mandante e gli esecutori}

Prima di giungere al 17 e 18 marzo 1938, giorni sui quali focalizzeremo la nostra attenzione, appare utile dare sommarie notizie sulle precedenti azioni belliche di bombardamento effettuate sul capoluogo catalano. L'8 ${ }^{\circ}$ Stormo iniziò queste attività l'8 dicembre 1937, ma non abbiamo l'indicazione del quantitativo di bombe sganciato in questa incursione. Fu poi la volta dei "Pipistrelli delle Baleari" che scaricarono 23.000 Kg di esplosivo colpendo nelle notti dell'11 (30 bombe da $100 \mathrm{Kg}$ ) e del 19 (6o bombe da

\footnotetext{
7 Stato Maggiore Aeronautica -Ufficio Storico - Operazione Militare Spagna (da ora in poi SMA-USOMS) - Cartella 51 - Sottocartella B. Contiene il "Diario Storico dell' $8^{\circ}$ Stormo"; SMA-US-OMS - Cartella 54 - Sottocartella "B". Contiene il "Diario Storico del XXV Gruppo Autonomo Bombardamento Notturno".
} 
$100 \mathrm{Kg}$ ) dello stesso mese e in quelle dell'8 gennaio 1938 (40 bombe da $50 \mathrm{Kg}$ ), del 12 (6o bombe da $50 \mathrm{~kg}$ ), del 16 (12 bombe da $250 \mathrm{~kg}$ ) e del 21 e 22 dello stesso mese (entrambe con 30 bombe da 100). Queste notti furono alternate con azioni diurne dei "Falchi delle Baleari" del 19 gennaio, dove tra Sagunto, Valencia, porto e depositi di Barcellona vennero lanciare 24 bombe da $250 \mathrm{Kg}, 48$ da $100 \mathrm{Kg}$ e 10 da $20 \mathrm{Kg}$, del 25 , con le formazioni che, al comando del Col. Rossanigo e del Magg. Lamanna, sganciarono 88 bombe da $100 \mathrm{Kg}$ e 44 da $20 \mathrm{Kg}$ su capannoni depositi di materiale, e del 30 gennaio, dove, sempre sul porto della capitale catalana, le due formazioni di 6 apparecchi ai comandi del Cap. De Prato e del Magg. Lamanna, ad intervallo di due ore, sganciarono 36 bombe da $250 \mathrm{Kg}$ e 24 da 20.

Solo il maltempo e alcuni ordini giunti direttamente da Roma, impedirono il continuo di questa attività bellica che riprese direttamente la notte tra il 16 e il 17 marzo. Già da questi primi dati ci si può ben rendere conto del fatto che Barcellona costituì un obbiettivo intensamente colpito dai bombardieri italiani.

Tab 1. - Bombardamenti aerei su Barcellona dall'8.12.1937 al 30.01.1938.

\begin{tabular}{|c|c|c|}
\cline { 2 - 3 } \multicolumn{1}{c|}{} & $\begin{array}{c}8^{\circ} \text { Stormo } \\
\text { (bombardamenti diurni) }\end{array}$ & $\begin{array}{c}\text { XXV Gruppo } \\
\text { (bombardamenti notturni) }\end{array}$ \\
\hline 8.12 .1937 & $?$ & $3.000 \mathrm{Kg}$ \\
\hline 11.12 .1937 & & $6.000 \mathrm{Kg}$ \\
\hline 19.12 .1937 & & $2.000 \mathrm{Kg}$ \\
\hline 8.01 .1938 & & $3.000 \mathrm{Kg}$ \\
\hline 12.01 .1938 & $11.000 \mathrm{Kg}$ & $3.000 \mathrm{Kg}$ \\
\hline 16.01 .1938 & & $3.000 \mathrm{Kg}$ \\
\hline 19.01 .1938 & & $3.000 \mathrm{Kg}$ \\
\hline 21.01 .1938 & $9.680 \mathrm{Kg}$ & \\
\hline 22.01 .1938 & $9.480 \mathrm{Kg}$ & $23.000 \mathrm{Kg}$ \\
\hline 25.01 .1938 & $30.160 \mathrm{Kg}$ & \\
\hline 30.01 .1938 & & \\
\hline Totale & & \\
\hline
\end{tabular}

8 A questo totale manca il dato del quantitativo di esplosivo lanciato l'8 dicembre 1937. 


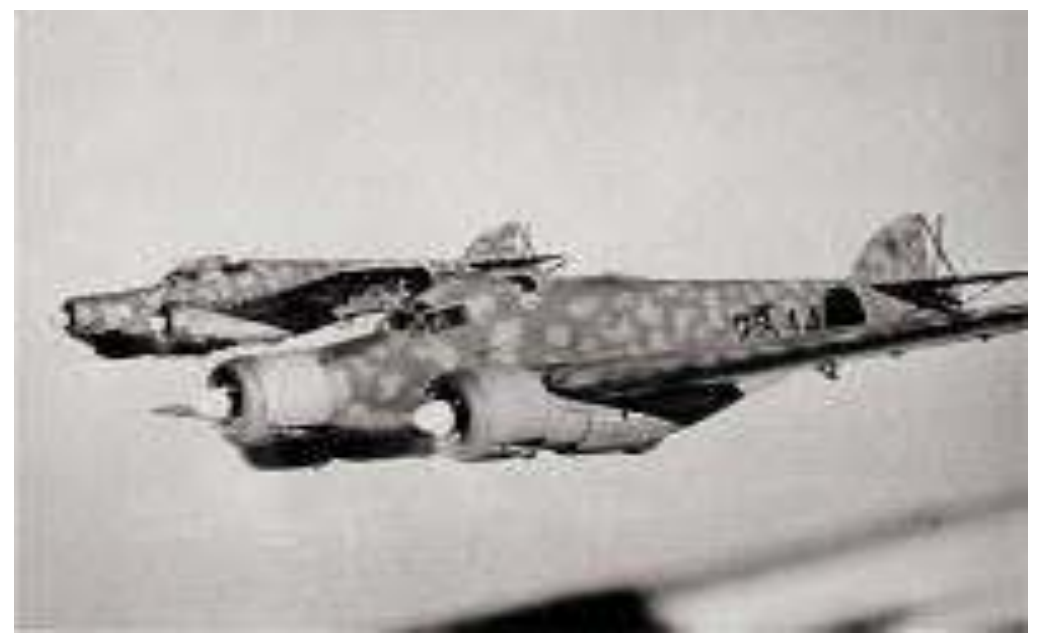

Fig. 1

Aereo SM.79 realizzato dalla Savoia-Marchetti in oltre 1200 esemplari, entrò in linea di volo nell'ottobre del $1934^{9}$.

Dall'8 dicembre 1937 al 30 gennaio 1938 possiamo contare, complessivamente, 7 notti di bombardamenti del XXV Gruppo Autonomo e 4 giorni di bombardamenti dell' $8^{\circ}$ Stormo, per oltre 50 tonnellate di bombe. Per avere una idea di cosa fosse possibile realizzare con i mezzi a disposizione in quel periodo, possiamo valerci di una descrizione che Ciano annotò sul suo diario. L'8 febbraio 1938, scrisse: «Ho ricevuto e dato al duce un rapporto di testimone oculare sul bombardamento recentemente fatto su Barcellona. Non ho mai letto un documento così realisticamente terrorizzante. Eppure erano soltanto $9 \mathrm{SM} 79$, e tutto il raid è durato un minuto e mezzo. Palazzi polverizzati, traffico interrotto, panico che diveniva follia: 500 morti, 1500 feriti. E' una buona lezione per il futuro. Inutile pensare alla protezione antiaerea ed alla costruzione di rifugi: unica via di salvezza contro gli attacchi aerei è lo sgombro delle città».10

Nove SM79 in un unico raid della durata di un minuto e mezzo: palazzi polverizzati, panico e 500 morti. Sono dei dati che occorre tenere a mente per valutare i bombardamenti del successivo marzo.

\footnotetext{
9 Il velivolo SM.79 aveva una apertura alare di 21,20 m, una lunghezza di 15,60 m e un'altezza di 4,60 $\mathrm{m}$. La sua capacità di trasporto era di 6 persone di equipaggio e fino a $1250 \mathrm{Kg}$ di esplosivo. L'autonomia di volo era stimata in $2300 \mathrm{Km}$. (Foto: Stato Maggiore Aeronautica - Fototeca Storica).

10 CIANO, Galeazzo, Diario 1937-1943, Milano, Rizzoli Editore, 1980, Giorno 8 febbraio 1938, p 95.
} 


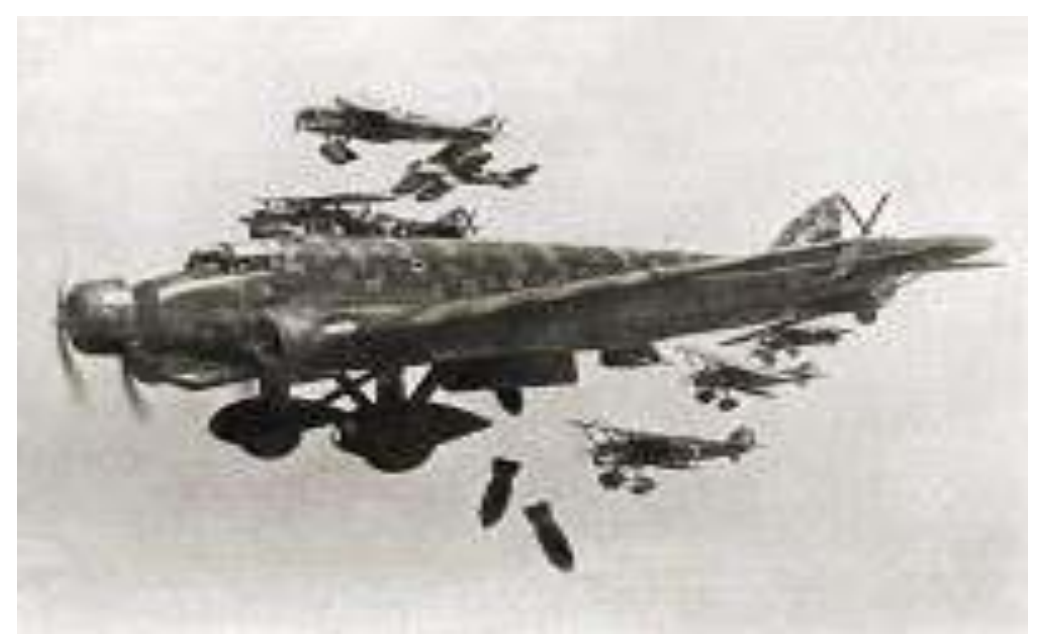

Fig. 2

Un bombardiere Savoia-Marchetti 81 in primo piano con una scorta di caccia Fiat Cr.32 che lo scortano nell'azione bellica.

Per la descrizione dei bombardamenti dei giorni 17 e 18 marzo 1938 faremo riferimento a quanto riportato nel diario storico dell' $8^{\circ}$ Stormo, integrandolo con la lettura di un altro importante documento: "Stralcio informazioni sugli effetti dei bombardamenti aerei dal $1^{\circ}$ gennaio 1938 al 29 marzo 1939"11 contenente informative e notizie radio raccolte dal Comando dell'Aviazione Legionaria delle Baleari e dallo Stato Maggiore della Jefatura del Aire.

Tab 2. - Bombardamenti aerei su Barcellona del 17 e 18 marzo 1938.

\begin{tabular}{|l|l|}
\hline \multicolumn{1}{|c|}{$\mathbf{1 7 . 0 3 . 1 9 3 8}$} & \\
\hline Efficienza bellica: & n. 11 SM.79; \\
\hline Attività bellica: & Vengono effettuati 3 bombardamenti, \\
& $\begin{array}{l}\text { ad intervallo di } 3 \text { ore, su magazzini e } \\
\text { depositi di Barcellona, da parte di } 3\end{array}$ \\
& formazioni composte rispettivamente \\
& da n. 6,5 e 5 apparechi; \\
\hline Comandanti delle & Col. Rossanigo; \\
formazioni: & Magg. Di Carlo; \\
& Cap. Balbo di V.; \\
\hline Ore di volo: & 43 ore e 50 minuti: \\
\hline Esplosivo lanciato: & 8 bombe da $250 \mathrm{~kg} ;$ \\
& 112 bombe da $100 \mathrm{~kg} ;$ \\
\hline
\end{tabular}

11 SMA-US-OMA - Cartella 34 - Sottocartella " $C$ ". 


\begin{tabular}{|c|c|}
\hline & 68 bombe da $20 \mathrm{~kg}$; \\
\hline Risultato dichiarato: & obbiettivi centrati. \\
\hline \multicolumn{2}{|l|}{ 18.03.1938 } \\
\hline Efficienza bellica: & n. 10 SM.79; \\
\hline Attività bellica: & $\begin{array}{l}\text { Viene effettuata una azione di } \\
\text { bombardamento su magazzini e } \\
\text { depositi di Barcellona da } 4 \text { formazioni } \\
\text { di } 3 \text { apparecchi ciascuna, agenti in } \\
\text { tempi separati. }\end{array}$ \\
\hline $\begin{array}{l}\text { Comandanti delle } \\
\text { formazioni: }\end{array}$ & $\begin{array}{l}\text { Ten. Piacentini; } \\
\text { Ten. Faralli; } \\
\text { Ten. Profumi; } \\
\text { Ten. Vanini. }\end{array}$ \\
\hline Ore di volo: & 27 ore e 50 minuti: \\
\hline Esplosivo lanciato: & $\begin{array}{l}24 \text { bombe da } 250 \mathrm{~kg} \text {; } \\
48 \text { bombe da } 100 \mathrm{~kg} \text {; } \\
30 \text { bombe da } 20 \mathrm{~kg} \text {; }\end{array}$ \\
\hline Risultato dichiarato: & obbiettivi colpiti in pieno - provocati diversi incendi. \\
\hline
\end{tabular}

Gli aerei dell' $8^{\circ}$ Stormo sganciarono, in due giorni, $25.960 \mathrm{~kg}$ di esplosivo sulla città di Barcellona. Ai bombardamenti diurni sopra riportati vanno aggiunti quelli notturni effettuati dai "Pipistrelli delle Baleari" la notte del 16 marzo: tre raid aerei con velivoli SM81 sganciarono un totale di circa $10.000 \mathrm{Kg}$ di bombe; e della notte del 17 marzo: ulteriori tre raid, sempre con SM81, dove lanciarono ulteriori $12.000 \mathrm{Kg}$ di esplosivo. In totale il bombardamento constò circa $47.960 \mathrm{~kg}$ di esplosivo in tredici raid aerei che si susseguirono giorno e notte, per quasi 48 ore. Paragonato al raid di cui ci ha dato illustrazione Ciano nel suo diario, dove un raid di un minuto e mezzo di 9 SM79 produsse 500 morti e 1500 feriti, i bombardamenti dei queste due giornate appaiono devastanti, così come il paragone con i circa $53.000 \mathrm{Kg}$ di bombe lanciate nel precedente periodo dall' 8 dicembre 1937 al 30 gennaio 1938, le circa 48 tonnellate sganciate in 48 ore appaiono di una intensità spaventosa. Per comprendere i danni prodotti, possiamo dare lettura allo "Stralcio informazioni sugli effetti dei bombardamenti aerei dal $1^{\circ}$ gennaio 1938 al 29 marzo $1939^{\prime 12}$ con l'avvertenza che il documento è basato unicamente su informatori del regime e su notizie radiofoniche

12 Ibidem. 
comunque non verificate al momento della loro acquisizione:

a. raccolte dai bollettini dallo Stato Maggiore della Jefatura dell'Aire:

- informativa del 23 marzo 1938:

È stata distrutta la fabbrica di armi "La Isalda" situata alla fine di viale di S. Juan. Si conosce che i recenti bombardamenti su Barcellona hanno causato enorme impressione fra gli elementi che frequentano l'Ambasciata rossa di Parigi. La popolazione è demoralizzata, i capi preoccupati e impressionati. Gli ultimi bombardamenti hanno colpito le fabbriche "Vulcano" e "Maquinista terrestre e marittima” le cui officine sono rimaste quasi completamente distrutte. Grandi danni si sono verificati nella stazione Nord (per la Francia). In uno dei bombardamenti effettuati recentemente fu colpito ed incendiato un importamene deposito di lubrificanti. Il Vice Console Americano in Barcellona riferisce che negli ultimi bombardamenti effettuati dall'Aviazione Legionaria fu colpito il porto, le zone industriali della città, i sobborghi del Nord, alcune fabbriche e due stazioni importanti ove sono stati causati danni ingenti.

- informativa del 26 marzo 1938:

...(omissis) Il giorno 17 marzo fu colpito e distrutto un treno che carico di militari si trovava in stazione pronto a partire. Inoltre è stata colpita la stazione del Nord che ha riportato ingenti danni.

b. raccolte dai bollettini della Radio Nazionale:

- 19 marzo 1938, ore 11.00:

L'incursione da parte di apparecchi nazionali ha causato ingenti danni ad opere militari. Durante il bombardamento è rimasto ucciso il Vice Console francese.

- 19 marzo 1938, ore 22.00:

Gli obbiettivi militari della capitale catalana sono stati oggetto nelle ultime 36 ore di otto bombardamenti da parte di apparecchi nazionali. I danni sono ingenti. La popolazione è demoralizzata.

c. raccolte dai bollettini del Comando Generale delle Baleari - Stato Maggiore:

- $1^{\circ}$ aprile 1938 , Risultato dei bombardamenti del 17 e 18 marzo su Barcellona:

Ci si informa che l'Hotel Colon e l'edifici adiacente, deve esistano gli Uffici del Banco Commerciale di Barcellona, sono stati in parte distrutti ed incendiati. Altre bombe caddero sul Banco di Vizcaya e sull'edificio del Consolato Francese. La stazione M.Z.A. è stata quasi totalmente distrutta e gravemente danneggiato il Mercato della Boqueria.

Una delle bombe che cadde nella Piazza della Catalogna provocò una grande fossa e penetrò a terra per alcuni metri.

Un'altra bomba cadde nella Ronda de San Antonio colpendo e distruggendo con 
i viaggiatori due carrozze tramviare e un autobus.

- $1^{\circ}$ aprile 1938, Effetti degli ultimi bombardamenti su Barcellona:

Secondo informazioni ricevute,...(omissis), furono colpiti e danneggiati gli edifici dei periodici "El Diluvio" e la "Pubblicidad" senza però distruggere le macchine ivi esistenti.

- $1^{\circ}$ aprile 1938 , Demoralizzazione prodotta dagli ultimi bombardamenti sopra Barcellona:

Ci si comunica che dopo gli ultimi bombardamenti effettuati i giorni 16, 17 e 18 marzo c.a. molti commercianti non aprono più le porte dei loro negozi mentre altri provvedono alle vendite solo per pochissime ore al mattino

- $1^{\circ}$ aprile 1938, Dettagli degli ultimi bombardamenti sopra Barcellona:

Negli ultimi bombardamenti effettuati dalla nostra Aviazione sopra Barcellona sono stati colpiti i seguenti edifici:

Hotel Colon, piazza di Catalogna;

un grande garage sulla strada di Muntaner;

il cinema Coliseum, via de Cortes;

il teatro Novedades, via de Caspe;

il teatro Barcellona, Rambla de Catalogna;

l'edificio della compagnia d'assicurazioni La Mutua, Rambla de Catalogna;

la caserma dell'Avenida Icaria.

- 6 aprile 1938, Effetti del bombardamento sopra Barcellona:

...(omissis) ci si comunica che nella via Pelayo caddero varie bombe distruggendo completamente molte case...(omissis)...colpiti risultano gli edifici della strada di Aragon...(omissis)...nella strada nuova della Rambla (antica Conde de Asalto) caddero varie bombe distruggendo completamente tre edifici...(omissis) La chiesa della Concezione (via de Argon) ed uno chalet vicino all'abitazione dove risiede Prieto.

- 11 aprile 1938, Effetti del bombardamento sopra Barcellona:

...(omissis) durante i bombardamenti sopra Barcellona:

gli edifici situati nella prima parte della strada di Cortes;

il tratto compreso tra le strade Balmes-Piazza Università-Palayo e Cortes;

la casa di proprietà di Pich e Pon, Piazza di Catalogna n. 9;

l'edificio di Manuel Arus - Gari;

Strada di Trafalgar vicino l'Arco di Trionfo;

Salone di San Giovanni;

Strada del Carmine;

Governo Civile;

Mercato di S. Antonio;

Via di San Paolo; 
Roger de Flor - Diputacion;

Paralelo;

Cortes - Rocafort.

Qualche ulteriore dato, sicuramente verificato in maniera più precisa rispetto alle dichiarazioni sopra riportate, ce lo fornisce direttamente il Governo della Catalogna Departament d'Interior - Relations Institucionals i Partecipaciò /Direcciò General de la Memoria Democratica con i lavori eseguiti principalmente da Josep Solè i Sabatè e Joan Villaroia. Tra il 1937 e il 1938 la capitale catalana contò: 1.816 morti, 2.719 feriti, 320 edifici totalmente distrutti, 547 parzialmente distrutti e 941 danneggiati, il tutto dovuto ad azione bellica diretta di bombardamento aereo, ovvero senza tenere conto di morti e danni temporalmente successivi ai bombardamenti, ma ad essi indirettamente imputabili. La città si difese con la realizzazione di 1.394 rifugi ${ }^{13}$.

Per concludere questo resoconto sui bombardamenti di Barcellona del 17 e 18 marzo 1937, possiamo valerci anche di uno sguardo ai quotidiani dell'epoca. Per poter procedere anche ad un confronto delle notizie da essi divulgate nei giorni 18 e 19 marzo 1938, prenderemo in considerazione una testata inglese e una italiana.

«The Times - London Edition», senza apparenti incertezze, il 18 marzo scrisse: «500 morti a Barcellona» informando in seguito: «[...] not aimed at military objects. They were aimed at a section of the old city where the poor live huddled together like rabbits in their warrens» ${ }^{14}$. Lo stesso quotidiano inglese, il seguente 19 marzo, titolò: «L'agonia di Barcellona. 600 Morti e 1.100 feriti». In questo secondo articolo, in particolare, possiamo leggere che Attlee, leader dell'opposizione laburista, sottolineò quanto gli attacchi sulla capitale catalana fossero terrorizzanti per la popolazione e non colpissero obbiettivi militari. ${ }^{15}$

Passiamo alla stampa italiana ${ }^{16}$. Il «Corriere della Sera» del 18 marzo $1938^{17}$ scrisse, in quinta pagina con la firma " $M$ ", un articolo intitolato «Il popolo di Barcellona chiede la resa», in cui si legge: «Iersera alle 22.15 gli aerei nazionali hanno fatto una

13 Disponibile al sito: URL: < http://barcelonabombardejada.cat/interactiu/ > [consultato il 17.03.2011].

14 The Times - London Edition, 18 marzo 1938, p. 16. "[...] non indirizzati a obbiettivi militari. Erano indirizzati a una parte della città vecchia dove i poveri vivono ammassati insieme come conigli nelle loro tane».

15 The Times - London Edition, 19 marzo 1938, p. 12.

16 GRASSIA, Edoardo, L'Aviazione Legionaria da bombardamento - Spagna 1936-1939 - Iniziare da stanotte azione violenta su Barcellona, cit., pp. 159-163.

Da un'analisi degli articoli di tre tra le principali testate giornalistiche italiane quali «Corriere della Sera», "ll Messaggero» e «ll Mattino» dei giorni 17-19 marzo 1938 e relativi ai bombardamenti della città di Barcellona, emerge una chiara riproduzione di "veline" imposte dal regime. Questo palesa il forte interesse che il Governo italiano ebbe relativamente a questa singola operazione bellica.

17 Corriere della Sera, 18 marzo 1938, p. 5. 
incursione sulla città. [...] sono stati bombardati le opere di difesa e i magazzini militari [...] l'Aviazione Nazionale ha continuato oggi a bombardare la capitale catalana e i centri militari circostanti [...] nell'azione delle 14.00 uno degli obbiettivi della incursione è stata la stazione ferroviaria, in quell'ora pulsante di traffico, con numerosi treni carichi di truppe e materiali in partenza per Lerida. [...] Benché limitata alla difesa e agli edifici di carattere militare l'azione delle Forze Aeree di Franco ha profondamente impressionato la popolazione di Barcellona che riunita per la via [...] chiede unanime la resa». Sempre il "Corriere della Sera" del 19 marzo $1938^{18}$, in sesta pagina, titola: «Nuovo pesantissimo bombardamento delle opere militari di Barcellona». «L'Aviazione di Franco ha di nuovo volutamente colpito i centri militari, le opere di difesa, gli edifici del Governo rosso [...] insieme alle bombe hanno lanciato anche manifesti invitanti alla resa per non incorrere in ancor più potenti bombardamenti aerei».

Appare giusto il caso di sottolineare che mentre il giornale inglese concentrò le sue notizie sul numero dei morti e feriti dovuti ai bombardamenti sottolineando il fatto che gli ordigni furono puntati sulla popolazione civile ormai terrorizzata, il giornale italiano concentrò la sua comunicazione sul fatto che gli obbiettivi colpiti furono tutti militari e, nel secondo articolo, diede una precisa indicazione circa la provenienza dei bombardieri: «L’Aviazione di Franco». Scorrendo le pagine dello stesso Corriere della Sera dei giorni immediatamente precedenti, spicca il fatto che sino ad allora, nella descrizione delle azioni aeree svolte in Spagna dall'Aviazione Legionaria, fu sempre posta in evidenza l’italianità dei piloti e dei mezzi aerei. Diversamente, per un chiaro motivo di moralità legata alle morti civili, che avrebbero probabilmente intaccato la sua immagine, il regime, in questa occasione, preferì non vantare tale paternità.

Per conoscere i veri artefici di ciò che accadde possiamo valerci di due documenti: il diario di Galeazzo Ciano e il messaggio "urgentissimo", in partenza da Roma il 16 marzo 1938. Dal diario di Ciano, giorno 20 marzo 1938 ${ }^{19}$ : «[...] stamani Perth (Ambasciatore inglese in Italia, ndr) $\mathrm{mi}$ ha consegnato un appunto per richiamare l'attenzione sui bombardamenti di Barcellona aggiungendo che ciò potrebbe creare uno stato d'animo ostile alla continuazione dei negoziati italo-britannici. Ho risposto che l'iniziativa delle operazioni è a Franco e non a noi: avremmo quindi potuto usare influenza moderatrice ma non assumere impegni [...] la verità sui bombardamenti di Barcellona è che li ha ordinati Mussolini a Valle (Capo di Stato Maggiore della Regia Aeronautica, ndr), alla Camera, pochi minuti prima di pronunciare il discorso

18 Corriere della Sera, 19 marzo 1938, p. 6.

19 CIANO, Galeazzo, Diario 1937-1943, cit., p. 115. 
sull'Austria. Franco non ne sapeva niente e ieri ha chiesto di sospenderli per tema di complicazione con l'estero. Mussolini pensa che questi bombardamenti siano ottimi per piegare il morale dei rossi, mentre le truppe avanzano in Aragona [...]. Quando l'ho informato del passo di Perth, non se ne è molto preoccupato, anzi si è dichiarato lieto del fatto che gli italiani riescano a destare orrore per la loro aggressività anziché compiacimento come mandolinisti. Ciò, a suo avviso (di Mussolini, ndr) ci fa anche salire nella considerazione dei tedeschi che amano la guerra totale e spietata».20

Dall'incontro, che a mio giudizio fu casuale, tra Mussolini, che doveva pronunciare un discorso sull'Anschluss atteso da tutta l'Europa perché avrebbe chiarito in maniera inequivocabile i futuri assetti di guerra, ed il Generale Valle, Capo di Stato Maggiore della Forza Armata aerea, che si trovava lì per un discorso già da tempo in programma, scaturì il seguente messaggio:

«PER GENERALE VELARDI (.)

INIZIARE DA STANOTTE AZIONE VIOLENTA SU BARCELLONA CON MARTELLAMENTO DILUITO NEL TEMPO (.)»21

20 In realtà, gli inglesi, attraverso la Government Code e Cipher School, furono in grado di decriptare le comunicazioni italiane, e in particolare della Regia Aeronautica, durante il conflitto civile spagnolo, tanto da essere a conoscenza in maniera dettagliata circa le operazioni di bombardamento su Barcellona effettuate dai bombardieri italiani di Palma di Maiorca, come messo in luce da SANTONI, Alberto, Ultra Intelligenze e macchine Enigma nella guerra di Spagna 1936 - 1939, Milano, Mursia, 2010, pp. 165-166.

21 SMA-US-OMS, Cartella 75. II documento è firmato V.. Presumibilmente fu quindi lo stesso Gen. Valle a firmare l'ordine impartito da Mussolini. Al Gen. Velardi, Comandante dell'Aviazione Legionaria, fu chiesto un violento bombardamento sul capoluogo catalano, senza nessuna ulteriore specificazione. 


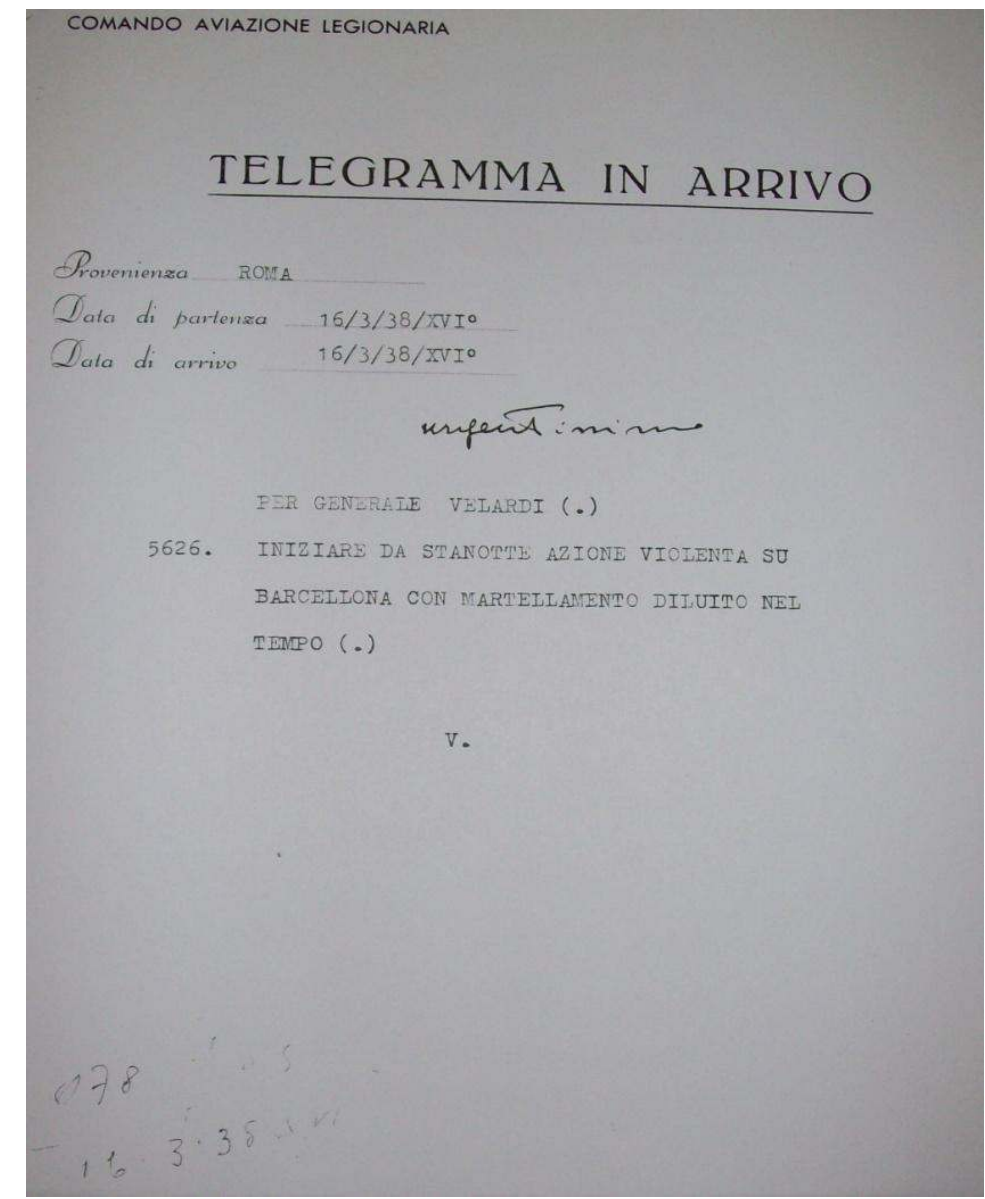

Fig 3

Telegramma “urgentissimo" 5626 partito da Roma il 16/03/1938 ${ }^{22}$

\section{Dall'Anschluss a Barcellona: i motivi di un bombardamento}

Rileggendo il passo del diario di Ciano sopra riportato: « [...] Mussolini pensa che questi bombardamenti siano ottimi per piegare il morale dei rossi, mentre le truppe avanzano in Aragona [...] $»^{23}$, potremmo affermare che i bombardamenti su Barcellona sopra illustrati fossero voluti per il desiderio di abbattere il morale della popolazione civile spagnola, per spezzare la resistenza repubblicana e per dare una svolta ad una guerra che si protraeva già da troppo tempo. Non sbaglieremmo di fronte a questa interpretazione, ma ci potrebbe essere anche un'altra lettura di questi avvenimenti che non faccia riferimento solo al conflitto civile spagnolo ma che allarghi il suo orizzonte all'imminenza della Seconda Guerra Mondiale con il suo preventivo gioco delle alleanze.

22 Ibidem.

23 CIANO, Galeazzo, Diario 1937-1943, cit., p. 115. 
Riprendiamo ancora una volta il diario di Ciano: «Quando l'ho informato del passo di Perth, non se ne è molto preoccupato, anzi si è dichiarato lieto del fatto che gli italiani riescano a destare orrore per la loro aggressività anziché compiacimento come mandolinisti. Ciò, a suo avviso [di Mussolini, ndr] ci fa anche salire nella considerazione dei tedeschi che amano la guerra totale e spietata» ${ }^{24}$. Sembrerebbe che il duce italiano fosse più interessato a salire nelle quotazioni dei tedeschi che al dover fronteggiare un problema diplomatico con gli inglesi. «Mi ha detto il duce [...] costruiremo trecento aeroplani al mese e ne avremo tremila di flotta. Bisogna stringere la cintola e armasi ${ }^{25}$ ». Ancora dal diario di Ciano apprendiamo che anche Mussolini era ben consapevole delle necessità militari dovute ad un probabile conflitto di grandi dimensioni.

Il 12 marzo 1938 Hitler, nell'ambito del suo noto progetto di costituzione del Terzo Reich, realizzò l'Anschluss ovvero l'occupazione militare dell'Austria. L'azione tedesca costituì sicuramente un evento importantissimo per la storia mondiale di quel particolare momento e dell'immediato futuro. Tale importanza, che probabilmente fu percepita con immediatezza tanto dagli statisti europei quanto dalla gente comune, la possiamo valutare attraverso la lettura dei quotidiani italiani che vanno dal 16 al 21 marzo 1938. In questi giorni le prime pagine furono dominate da articoli relativi all'azione tedesca, al conseguente discorso che Mussolini fece alla Camera e, successivamente, agli effetti suscitati dalle parole del duce italiano all'estero ${ }^{26}$. Le parole di Mussolini relative all'Anschluss erano attese in tutta Europa. Da queste, infatti, si sarebbe chiarita definitivamente la posizione italiana nel possibile futuro assetto delle alleanze militari: contenere e condannare l'azione tedesca con un conseguente avvicinamento alla Gran Bretagna e riposizionare le quattro divisioni del Regio Esercito sul Brennero, come in occasione del precedente putsch nazista o suggellare definitivamente l'amicizia con Hitler? L'ultimo discorso alla Camera del duce risaliva al precedente 11 dicembre 1937, in occasione dell'uscita dell'Italia dalla Società delle Nazioni. Per tre mesi non si sentì la necessità di nuovi discorsi fino all'annessione dell'Austria da parte della Germania, che il Capo del Governo affrontò con certa importanza.

Immediatamente dopo l'Anschluss, l'Aviazione Legionaria italiana - come visto nei diari storici dei reparti interessati - colpì ripetutamente la città di Barcellona. Abbiamo

Ibidem.

Ibidem, p. 72.

GRASSIA, Edoardo, L'Aviazione Legionaria da bombardamento - Spagna 1936-1939 - Iniziare da stanotte azione violenta su Barcellona, cit., p. 159. 
già osservato, ritrovando la notizia nel diario di $\mathrm{Ciano}^{27}$, che fu lo stesso Mussolini poco prima di entrare alla Camera per il suo atteso discorso - a riferire al Generale Valle di procedere con il bombardamento della città catalana, approfittando del fatto che lo stesso generale si trovava alla Camera per illustrare lo stato della Regia Aeronautica.

Così come afferma anche lo storico Coverdale, l'impatto dell'Anschluss provocò una dura reazione da parte del duce. Una parte dell'opinione pubblica aveva esaltato la tattica (e l'astuzia) con la quale Hitler aveva pianificato la sua "entrata trionfale" in Austria; Mussolini, non volendo essere da meno, organizzò una rapida manovra militare a grande scala: per motivi di sicurezza inviò al confine del Brennero numerosi mezzi blindati, mentre poco dopo diede l'ordine di attaccare Barcellona con un raid aereo (una manovra giustificabile perché in un teatro di guerra), per dimostrare la qualità dell'apparato militare italiano. Occorreva dar una prova di forza e di temperamento davanti alle altre nazioni europee, ma soprattutto era necessario far arrivare una chiaro messaggio a Hitler con la volontà di «salire, così, nelle sue considerazioni» 28 .

Hitler non solo aveva compiuto un'azione spregiudicata ma aveva anche dimostrato di non temere lo scoppio di un nuovo conflitto mondiale. Il Duce da parte sua, aveva sia mobilitato l'esercito che dato l'ordine di attacare: «la crudeltà di Mussolini può essere in parte spiegata con il suo bisogno di compensazioni psicologiche per l'Anschluss: era stato costretto ad essere passivo spettatore [...] e malgrado il suo atteggiamento di spavalda impudenza ostentato per scopi propagandistici, si era sentito profondamente umiliato di fronte all'opinione pubblica interna e internazionale ${ }^{29}$ ». Con ampie falcate, così come lo abbiamo visto più volte nei filmati dell'epoca, si diresse verso l'ingresso della Camera per annunciare all'Europa che «le due Nazioni [Italia e Germania, ndr] la cui formazione unitaria è stata parallela nel tempo e nei modi, unite come sono da una concezione analoga della politica della vita, possono marciare insieme per dare al nostro travagliato continente un nuovo equilibrio, che permetta finalmente la pacifica e feconda collaborazione di tutti i popoli» ${ }^{30}$. Un discorso molto forte, che non solo dichiarò le intenzioni italiane circa le definitive alleanze future, ma che anche compromise il

27 CIANO, Galeazzo, Diario 1937-1943, cit., p. 115.

28 Sempre al proposito di «salire nelle considerazioni dei tedeschi» attraverso dimostrazioni di potenza bellica, possiamo anche fare riferimento alla visita ufficiale di Hitler in Italia del 13 maggio 1938. L'evento fu evidentemente ritenuto molto importante visto che si stava preparando già dall'ottobre del 1937. Durante la visita, che durò ben sette giorni, oltre alle città di Roma, Napoli e Firenze, si inscenarono parate militari e soprattutto dimostrazioni di mezzi e azioni belliche a Napoli per la Regia Marina, a Furbara (nord di Roma) per la Regia Aeronautica e, poco lontano, a Santa Marinella, per il Regio Esercito.

29 COVERDALE, John F., I fascisti italiani nella guerra di Spagna, cit., p. 324.

30 Disponibile al sito URL: < http://www.dittatori.it/discorsimussolini.htm > [consultato in data 17.03.2011]. 
fascismo ad un maggior impegno nel panorama internazionale. Con queste parole già nella mente, il duce si incontrò con il Generale Valle, già elevato al comando della Forza Armata aerea e di quei bombardieri schierati alle Baleari, a poca distanza da Barcellona. Dall'incontro scaturì la decisione di dimostrare la qualità dell'impegno italiano in Spagna e la forza della sua aviazione; poche ore dopo, il Sottosegretario dell'Aeronautica, inviò un telegramma al Generale Velardi con il quale impartì l'ordine di «iniziare da stanotte azione violenta su Barcellona con martellamento diluito nel tempo»31. Un ordine, questo, firmato " $V$. ". In precedenza, molte azioni di bombardamento o di cessazione della attività belliche furono impartite direttamente da Ciano e da Mussolini ai comandanti in Spagna, saltando qualsiasi canale gerarchico. Una procedura adottata spesso per non creare delle autorità intermedie tra Mussolini e la base. Questa volta, invece, l'ordine fu impartito attraverso il Generale Valle e la variazione della procedura appare avvalorare la tesi che l'ordine fu impartito per il casuale incontro. Il martellamento sarebbe poi stato interrotto solo su ordine diretto del Generale Franco ${ }^{32}$.

Se questa fu la sequenza degli avvenimenti, Ciano, come già visto ancora attraverso il suo diario33, nel colloquio con l'ambasciatore inglese, ne operò una inversione: alla richiesta di chiarimenti di Lord Perth, infatti, rispose alterando la realtà dei fatti: come artefice dell'iniziativa dei bombardamenti fu indicato il Generale Franco. ${ }^{34}$ Possiamo ipotizzare che questo rovinoso episodio storico fu in realtà una conseguenza imprevista $\mathrm{e}$ casuale rispetto all'invasione tedesca dell'Austria. Possiamo dire che questa azione bellica fu, tra l'altro, un messaggio non scritto che Mussolini volle inviare a Hitler per dare dimostrazione che una alleanza con l'Italia era, militarmente parlando, una "sorta di buon affare”.

Possiamo concludere che, probabilmente, se il Generale Valle non avesse avuto il compito di illustrare lo stato della Regia Aeronautica alla Camera proprio quel giorno ed a quell'ora, l'ordine di bombardare così pesantemente la città di Barcellona forse non sarebbe stato emanato. Questa possibilità, in particolare, ci appare avvalorata anche tenendo in considerazione che le «decisioni più importanti erano affidate al duce; molte erano prese sotto lo stimolo del momento ${ }^{35}$ ». E' una possibile chiave di lettura di questo drammatico episodio che può essere aggiunta a quelle già illustrate ed inerenti alla volontà di dare una accelerazione al cedimento del crollo della fazione

\footnotetext{
31 SMA-US-OMS - Cartella 75. Telegramma del 16 marzo 1938.

32 SMA-US-OMS - Cartella 75. Telegramma del 18 marzo 1938.

33 CIANO, Ciano, Diario 1937-1943, cit., giorno 20 marzo 1938, p. 115.

34 GRASSIA, Edoardo, L'Aviazione Legionaria da bombardamento - Spagna 1936-1939 - Iniziare da stanotte azione violenta su Barcellona, cit., pag. 159 e ss.

35 COVERDALE, John F., I fascisti italiani nella guerra di Spagna, Bari, Laterza, 1977, p. 323.
} 
repubblicana ${ }^{36}$.

Relativamente a qualsiasi proposta di lettura, occorre comunque rifarsi a Orwell, che alla Catalogna ha dedicato un omaggio, nel suo asserire che: «attenzione alla mia partigianeria, ai miei errori di fatto e alla distorsione inevitabile causata dal mio aver visto un angolo degli eventi. E attenzione alle stesse identiche cose nel leggere qualsiasi altro libro su questo periodo nella guerra civile di Spagna» 37 .

\section{Bibliografia}

BROWN, Harry, La guerra civile spagnola, Bologna, Il Mulino, 2000.

CIANO, Galeazzo, Diario 1937-1943, Milano, Rizzoli, 1980.

COVERDALE, John F., I fascisti italiani nella guerra di Spagna, Bari, Laterza, 1977.

DOUHET, Giulio, Il dominio dell'aria e altri scritti, Roma, Aeronautica Militare Ufficio Storico dello Stato Maggiore, 2002.

GRASSIA, Edoardo, L'Aviazione Legionaria da bombardamento - Spagna 19361939 - Iniziare da stanotte azione violenta su Barcellona, Roma, IBN Editore, 2009.

ORWELL, George, Omaggio alla Catalogna, Milano, Il Saggiatore, 1964.

PELLICCIA, Antonio, «Riflessioni storiche sul potere aereo», Aeronautica, n. 3, Marzo 2007, pp. 8-11.

SANTONI, Alberto, Ultra Intelligenza e macchine. Enigma nella guerra di Spagna 1936-1939, Milano, Mursia, 2010.

\section{Sitografia}

http://www.cronologia.leonardo.it/storia/a1943.htm

http://www.barcellonabommardejada.cat

http://www.dittatori.it/discorsimussolini.htm

\section{Fonti d'archivio}

Stato Maggiore Aeronautica - $5^{\circ}$ Reparto - Ufficio Storico - Fondo "Operazione Militare Spagna - OMS” (SMA-US-OMS).

37 ORWELL, George, Omaggio alla Catalogna, Milano, II Saggiatore, 1964, p. 279. 


\section{* L'autore}

Edoardo Grassia, laureato in Sociologia (V.O.) presso l'Università "La Sapienza" di Roma, è ora studente del corso di laurea in Storia presso la stessa università. Si è occupato dell'Aviazione Legionaria da Bombardamento, da cui è nata la pubblicazione: L'Aviazione Legionaria da bombardamento (Spagna 1936-1939) - Iniziare da stanotte azione violenta su Barcellona, Roma, IBN Editore, 2009. Svolge regolarmente attività di studio e di ricerca in ambito storico e storico-militare.

URL: < http://studistorici.com/progett/autori/\#Grassia >

\section{Per citare questo articolo:}

GRASSIA, Edoardo, «Barcellona, 17 e 18 marzo 1938», Diacronie. Studi di Storia Contemporanea: Spagna Anno Zero: la guerra come soluzione, 29/07/2011,

URL:<http://www.studistorici.com/2011/07/29/grassia1_numero_7/ >

\section{Diacronie Studi di Storia Contemporanea $\beta$ www.diacronie.it}

Risorsa digitale indipendente a carattere storiografico. Uscita trimestrale. redazione.diacronie@hotmail.it

Comitato di redazione: Marco Abram - Giampaolo Amodei - Jacopo Bassi - Luca Bufarale - Alessandro Cattunar - Alice De Rensis Barbara Galimberti - Deborah Paci - Fausto Pietrancosta - Martina Sanna - Matteo Tomasoni - Luca Zuccolo 